\title{
Design of a High Precision, Wide Ranged ANALOG ClOCK GENERATOR WITH FIELD Programmability USINg Floating-gate TRANSISTORS
}

\author{
Garima Kapur \\ kapur.garima@gmail.com \\ Department of Physics and Computer Science, \\ Dayalbagh Educational Institute, Dayalbagh, Agra, INDIA \\ C.M Markan \\ cm.markan@gmail.com \\ Department of Physics and Computer Science, \\ Dayalbagh Educational Institute, Dayalbagh, Agra, INDIA \\ V. Prem Pyara \\ Department of Electrical Engineering, \\ Dayalbagh Educational Institute, Dayalbagh, Agra, INDIA
}

\begin{abstract}
This paper presents a circuit of a high-precision, wide ranged, analog clock generator with on-chip programmability feature using Floating-gate transistors. The programmable oscillator can attain a continuous range of time-periods lying in the programming precision range of Floating Gates. The circuit consists of two sub circuits: Current Generator circuit and Wave Generator circuit. The current of current generator circuit is programmable and mirrored to the wave generator to generate the desired square wave. The topology is well suited to applications like clocking high performance ADCs and DACs as well as used as the internal clock in structured analog CMOS designs. A simulation model of the

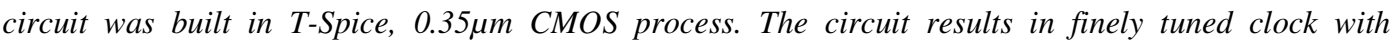
programmability precision of about 13bit [1]. Simulation results show high amount of temperature insensitivity $\left(0.507 \mathrm{~ns} /{ }^{\circ} \mathrm{C}\right)$ for a large range of thermal conditions. The proposed circuit can compensate any change in temperature. The circuit design can be operated at low supply voltage i.e., $1 v$.
\end{abstract}

\section{KEYWORDS}

Square wave generator, floating gate FET, field programmability

\section{INTRODUCTION}

There are many oscillators like crystal oscillators; Novel RC oscillator [2], RC active frequency Oscillator [3], etc., have been developed, to accomplish the need of internal clock for synchronization between cells in VLSI circuit designs. However due to large size limitation, opamp offsets and complicated designs respectively, many digitally on-chip trimmed RC oscillator 
circuit designs [4], [5], [6] have also been developed. These clock generators are temperature insensitive and produce accurate clock which can be digitally trimmed using array of resistors or weighted capacitors.

With time the need for high speed data transmission and accuracy increases in the system designs. Hence a highly flexible and high precision clock generator is required to optimize for the next generation of wired or wireless network equipment that demands highly accurate clock generator and distribution for robust high speed transmission. Thus the research went in some other direction; clock generator using organic thin film transistors (OTFT) and inverters with bootstrapped transistors have been developed [7]. However it generates very low frequency clock. The wide and continuous ranged, accurate clocks are required in optical networks, mixed signal circuits, ATE, medical imaging and automated test equipments. Solutions proposed for such clock generation in recent years include usage of large devices and careful layout or some trimming and calibration techniques.

In this paper we use floating gate p-channel field effect transistor (FG-pFET) as a method for field programmable trimming of clock frequencies. The design can generate fine-tuning of the clock. In FG-pFET, by changing the charge at the gate we can program the variable frequency range. Tunable FG-pFET resistor offers good precision while passive resistors implemented using polysilicon, diffusion or well strips in CMOS technology exhibit around $0.1 \%$ matching accuracy and around 30\% tolerance [8]. As well as tunable FG-pFET resistor will acquire less chip area as compared to other on-chip digital trimming circuit designs.

In section 2, we give the circuit diagrams of floating gate transistor, its programming and simulation model of tunable FG-pFET resistor. We perform the simulations for output clock generation and explained the design and programming methodology of the proposed circuit design. In section 3 and 4, we compared our proposed clock generator with digitally trimmed clock generator [4]. In section 5 and 6 technique used for on-chip programming and topology to widen frequency range of the output clock are briefly described. The temperature analysis of the proposed circuit is also shown in section 7.

\section{Proposed Circuit}

This paper presents a simple RC Oscillator circuit based on the charge variation at the floatinggate of a FG-pFET which provides the flexibility of achieving a continuous range of resistance value, which in turn provides variable current required to generate the output clock. The clock generated can be precisely tuned with approximately 13 bit resolution [1]. The continuous, finetuned and wide ranged clock can be obtained. Compensation of variations due to change in temperature can be obtained by just tweaking the floating gate voltage of FG-pFET according to the specified temperature.

\subsection{Floating-gate Transistor}

Before introducing the basic floating gate clock generator, a brief discussion of floating gate transistors is beneficial. Though there are different types and variations of floating gate transistors, their basic construction and operation remain the same. Floating gate transistors are usually MOS transistors wherein memory is stored in the form of charge trapped on floating gate, affecting its threshold voltage. Since the gate is electrically isolated due to oxide completely surrounding it, the charge on the gate is fixed and is responsible for establishing the 
amount of current flowing through the transistor. While the charge on the gate will not change on its own, that the processes such as UV photo injection, Fowler-Nordheim tunneling, and Hotelectron injection can modify that amount of charge. The last two are the primary means of programming floating-gate transistors to precise currents [9]. Once the desired charge is pushed onto the floating gate, it can be utilized to provide a constant bias to other transistors in a design, from which a precisely tuned clock can be generated.

Figure.1 shows the structure of a floating gate transistor. Due to their architecture, floating gate transistors are small, easily manufactured in CMOS processes, and provide simple postfabrication programmability; characteristics which make them very useful in designing clock generator circuits.

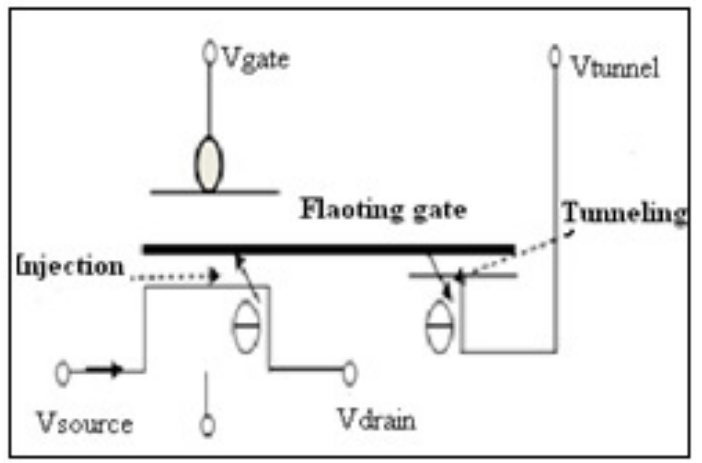

Figure 1: Structure of floating-gate transistor [9]

Programming ability of the oscillator is obtained by modifying the charge stored on the floating gate. There are two programming topologies: direct and indirect programming. However, indirect programming provides real time applications to the design. It removes the necessity of a separate programming phase and an operational phase. As, in indirect programming, one pFET is connected to the programming structure while the source and drain of the other transistor are connected to the respective circuit (proposed circuit). The first pFET is programmed with hot-electron injection and tunneling. Since the charge on this "programmer" pFET (Mp) is modified, the current of the other transistor (the "agent") (Ma) will also be set as explained in figure2. With this programming technique, multiple FETs can share a common floating gate, and hence output range can be extended as explained in section 6 .

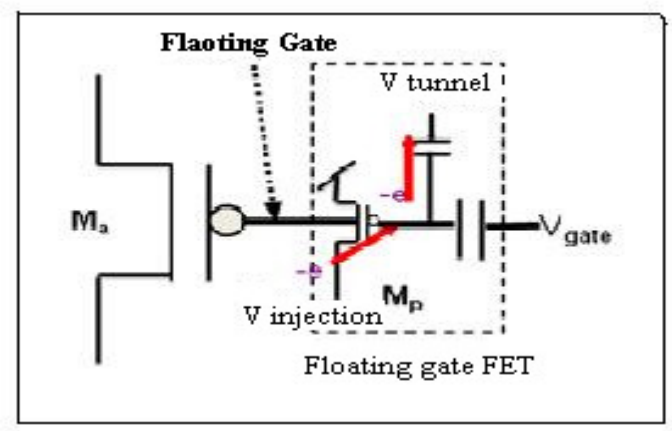

Figure 2: Indirectly programmed FG-pFET [10] 


\subsection{Tunable FG-pFET Resistor}

The fundamental requirement to operate a single MOS transistor in the triode region as a linear resistive element is to suppress its nonlinearities by applying a function of the input signal to its gate [11] and/or its body [12]. The three principal nonlinearities in the drain current of a longchannel transistor in the triode region are identified as the body effect, the mobility degradation, and the fundamental quadric component due to the common-mode of the drain and source voltages [13]. Therefore to attain linearization in CMOS resistor a common mode and large voltage is employed at the gate. However, this technique does not completely eliminate the body effect and the mobility degradation. The gate linearization is achieved by using model from [13].

Since the transistor does not need to be disconnected from the circuit to program it, the switch count is reduced, resulting in fewer parasitic and better overall performance [10]. With the reference from [14] we have implemented FG-pFET indirectly programming circuit. Voltage dependent current sources and programmable PMOS have been used for tunneling and hot-electron injection to the floating gate as shown in figure3. For injection drain to source voltages of the programming PFET is varied and for tunneling $\mathrm{V}_{\text {tun }}$ is varied. Thus charge at the floating gate voltage changes and consecutively range of resistance can be obtained across the transistor.

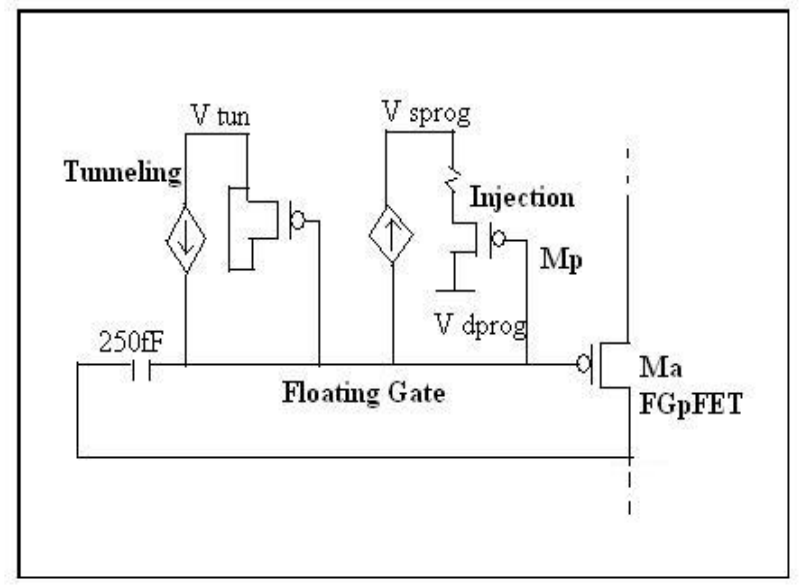

Figure 3. T-Spice 0.35um CMOS process Simulation Model of indirectly programmed tunable FG-pFET resistor

The simulation model of the indirectly programmed tunable FG-pFET was built in T-Spice, 0.35um CMOS process. The characteristics have been obtained from the simulation model. The hot-electron injection and tunneling currents have been plotted with respect to the floating gate voltage, as shown in figure 4. Due to these injection and tunneling currents variation which in turn, varied by using $\mathrm{V}_{\text {tun }}, \mathrm{V}_{\mathrm{s}_{-} \text {prog }}$ and $\mathrm{V}_{\mathrm{d}_{-} \text {prog }}$ in the simulation model in figure 3, the floating gate voltage changes and hence the tunable resistance range can be obtained. Thus, the tuning range of FG-pFET resistor with change in floating gate voltage has been catered in figure 5. 


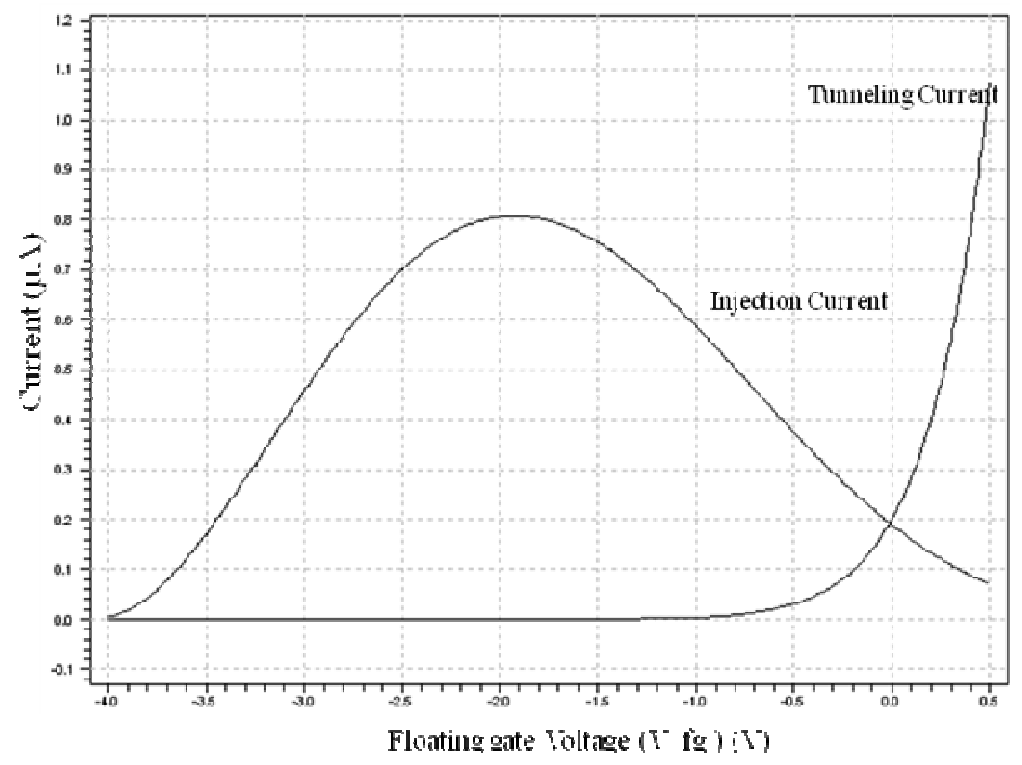

Figure 4: Characteristic currents of the indirectly programmed Floating gate transistor

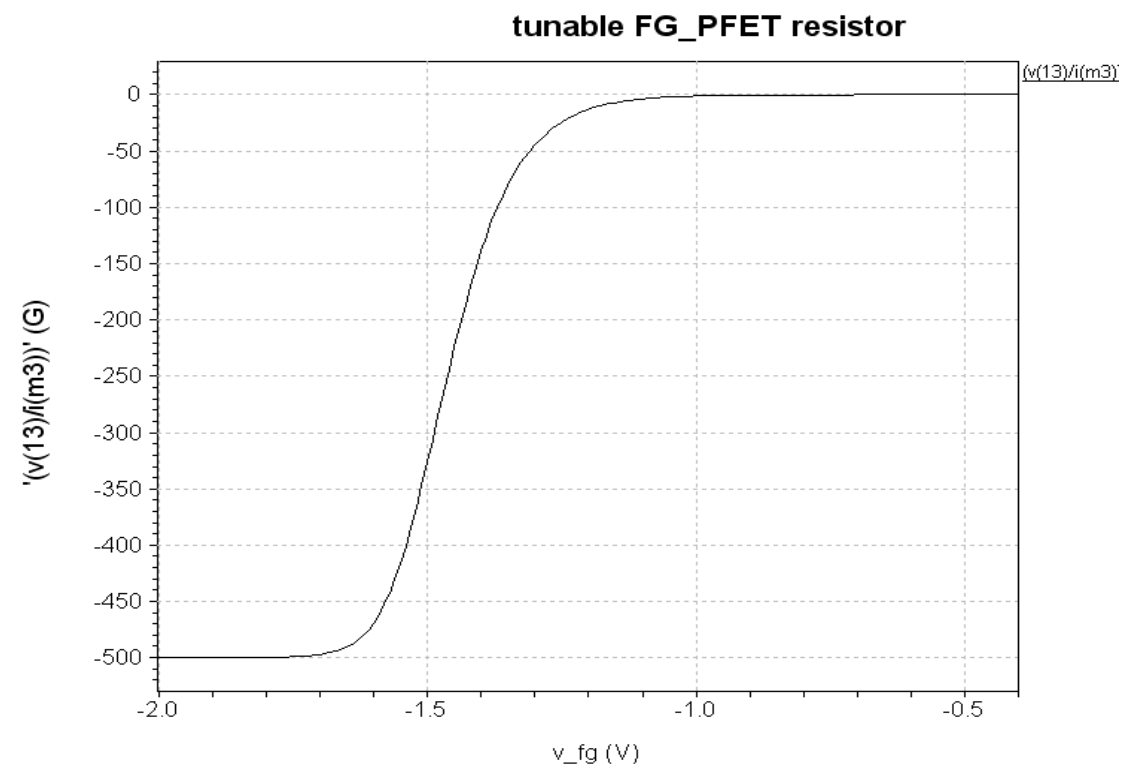

Figure 5: Tunable FG-pFET resistor range with respect to varying floating gate voltage, obtained from its simulation model implemented in T-Spice 0.35um CMOSS process.

\subsection{FG-pFET Programmable Clock Generator}

The proposed Clock generator circuit using floating gate pFET as shown in Figure 6 consists of a current source sub-circuit and a wave generator sub-circuit [4]. The current generator is a kind of current mirror whose current is controlled by FG-pFET. The current from the current generator sub-circuit is mirrored to the wave generator sub-circuit. The wave generator uses a switch (nmos) to control the charging and discharging function of the capacitance to generate a triangular wave. Then this triangular wave is transformed to spikes by the combination of invertors, which in turn feed to the clock input of the D flip-flop where, input of the D flip-flop 
is shorted with the inverted output of the D flip-flop to generate the square wave from the spikes.

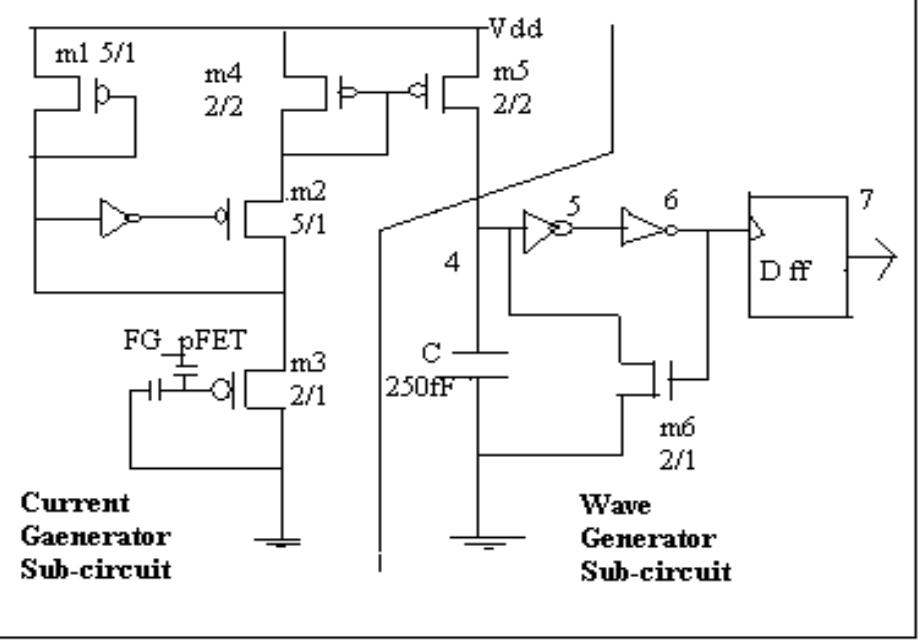

Figure 6: Proposed Clock generator circuit design simulated using T-Spice 0.35um CMOSS process.

In the current source sub-circuit, a FG-pFET is employed which is programmed by indirect programming as explained in the last section. The continuous ranges of resistance value are obtained to generate varying current which in turn mirrored to the wave generator circuit. Thus, continuous and finely tuned square wave is generated.

\subsection{Design Methodology}

The architecture consists of the two sub-circuits as shown in figure 6:

Wave generator circuit:

Voltage at the capacitor $\mathrm{C}$ depends on the current transferred from current mirror (i.e. current at node 4)

$$
V_{c}=\frac{I \times \Delta t}{C}
$$

Threshold of invertors, turn on the low impedance NMOS switch to discharge the capacitor to zero voltage. Hence, period of the triangular voltage waveform is:

$$
T_{c}=\Delta t=\frac{C \times \Delta V_{c}}{I}
$$

Current generator circuit:

A constant current source is built using current mirror. The current is generated from a PFET (source follower). A feedback circuit always stabilizes the voltage above floating-gate PFET (V1). Therefore, the floating gate PFET will decide the current.

$$
I_{F G-P F E T}=\frac{\mu C_{o x} W}{L}\left(V_{g s}-V_{t h}\right) V_{1}, V_{1}=V_{d s}
$$


This current is substituted to get the period of charging/ discharging of capacitor as:

$$
T_{c}=\frac{C \Delta V_{c} L}{\mu C_{o x} W\left(V_{g s}-V_{t h}\right) V_{1}}
$$

The threshold voltage of the floating-gate PFET decreases/increases with increase/decrease of floating gate voltage (to maintain transistor in linear region). Hence, the resistance of the transistor changes which in turn changes the period of charging/discharging of the capacitor.

$$
T_{c}=R_{F G-P F E T} C \times \frac{\Delta V_{c}}{V_{1}}
$$

However, the period of the output clock is two times of this period (Tc). Therefore the output square wave generated can be tuned by just varying the resistance of the floating-gate PFET.

\subsection{Programming Methodology}

Using floating gate transistor synapse as shown in figure 3 we have implemented the indirect programming of FG-pFET (M3 in figure 6). Injection requires $>1.5 \mathrm{~V}$ drain-to-source voltage across the injection transistor $\left(\mathrm{M}_{\mathrm{p}}\right.$ in figure 3 ) while tunneling requires $>8 \mathrm{~V}$ across the tunneling terminal for $0.35 \mu \mathrm{m}$ CMOS process.

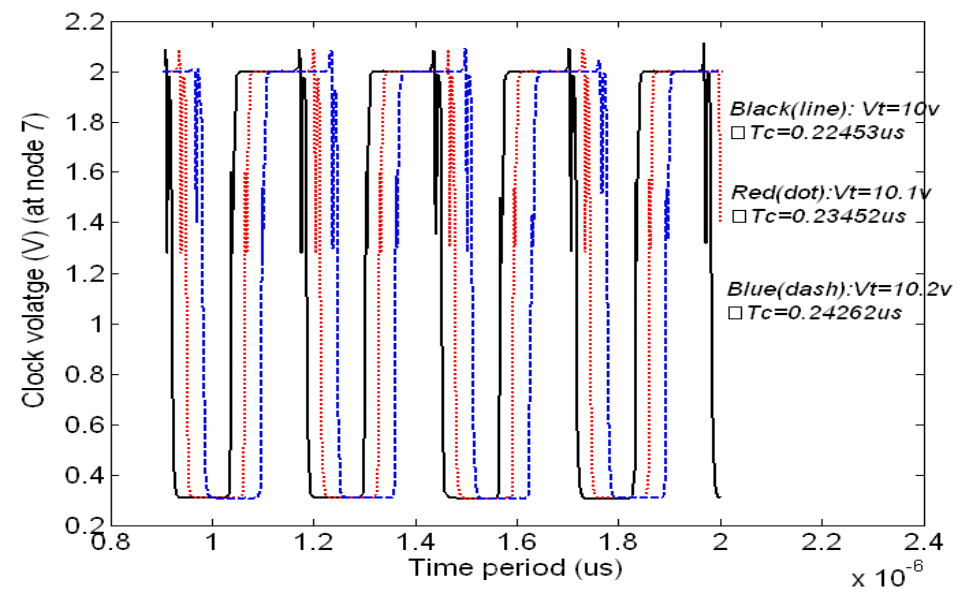

Figure 7: Variation in output clock with respect to tunneling voltage $\left(\mathrm{V}_{\mathrm{t}}=\mathrm{V}_{\text {tun }}\right)$

The following two graphs will represent the change in output clock with change in tunneling voltage and with change in drain to source voltage in programmable PFET i.e. changes due to tunneling and injection respectively.

With increasing tunneling voltage, threshold voltage of floating-gate pFET $\left(\mathrm{M}_{\mathrm{a}}\right.$ in figure 3$)$ increases and thus the resistance, this sequentially increases the time period of the output clock (as shown in figure 7). Whereas when the drain-to-source voltage of programmable PFET decreases, i.e. injection decreasing, threshold voltage increases and hence the period of the output clock (as shown in figure 8). 


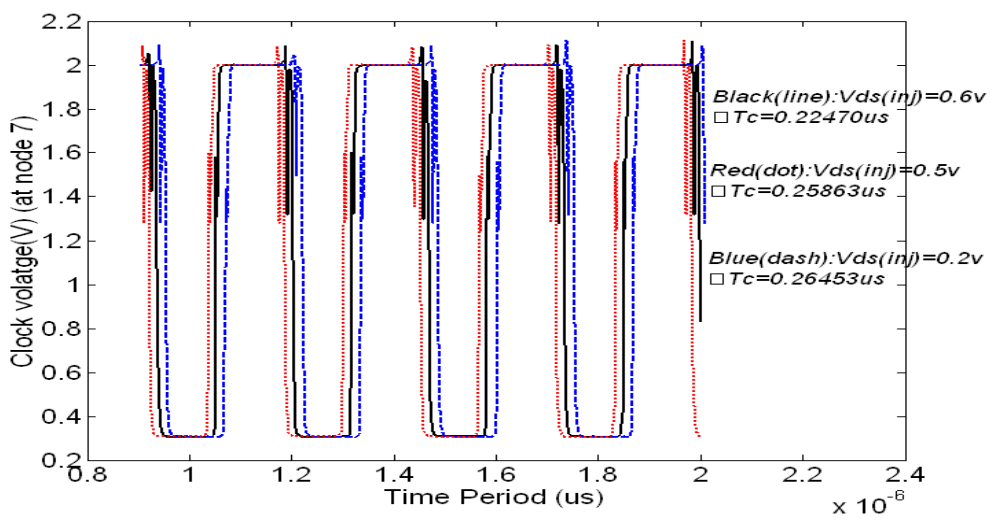

Figure 8: Variation in output clock with respect to injection voltage $\left(\mathrm{Vds}(\mathrm{inj})=\mathrm{V}_{\mathrm{d}_{-} \text {prog }}-\mathrm{V}_{\text {s_prog }_{\text {prog }}}\right)$

\subsection{Simulated Results}

Figure 9 first wave shows the voltage across the capacitor (V4) (node 4 in figure 6). It shows the charging and discharging of the capacitor using NMOS switch. Fig8 middle wave shows the invertors output (V6) (node 6). The input D of the flip flop is shorted with its complemented output. Thus, Figure 9 last wave showing the output square wave (output clock) obtained from the D flip-flop (V7) (node 7).

The square wave generated has been kept at low frequencies by adjusting the W\&Ls of transistor used and by keeping high capacitor(C) value $(250 \mathrm{fF})$. As at high frequency like $20 \mathrm{MHz}$ very high distortion in the clock has been observed. While at low frequency 1 to $8 \mathrm{MHz}$ clear and better clock is generated as shown in figure 9 .
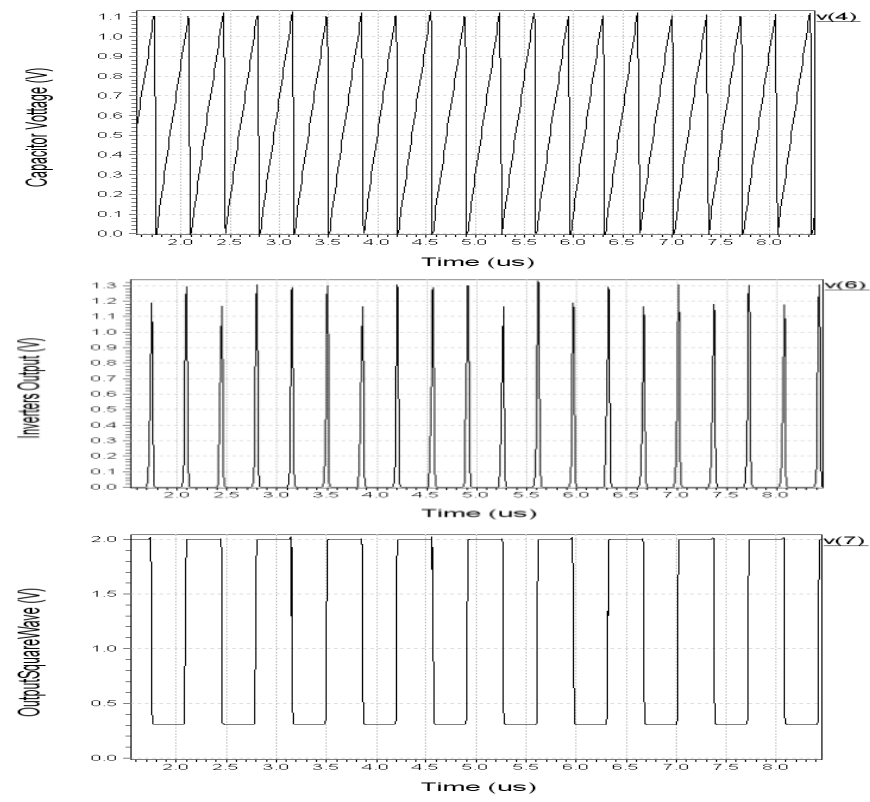

Figure 9: The simulated output waveforms (capacitor C output, inverters output and the D flipflop output sequentially represented) obtained from proposed simulation model on T-spice 0.35um CMOSS process. 


\section{Comparative Trimming Results}

Table 1. Digitally trimmed resistor array RC oscillator inspired from [5]

\begin{tabular}{|c|c|c|c|}
\hline Switching & Resistance (K) & $\begin{array}{c}\text { Time Period } \\
(\mathbf{u s})\end{array}$ & Frequency (MHz) \\
\hline 1111111 & 47.8 & 0.15 & 6.67 \\
\hline 0111111 & 75.9 & 0.18 & 5.55 \\
\hline 0011111 & 99.98 & 0.2 & 5 \\
\hline 0001111 & 120.4 & 0.22 & 4.76 \\
\hline 0000111 & 137.89 & 0.26 & 3.33 \\
\hline 0000011 & 154.26 & 0.3 & 2.63 \\
\hline 0000001 & 167.83 & 0.38 & 2.63 \\
\hline 0000000 & 175.3 & 0.45 & 2.22 \\
\hline 1000000 & 151.02 & 0.3 & 3.33 \\
\hline 1100000 & 125.35 & 0.25 & 4 \\
\hline 1110000 & 105.35 & 0.2 & 5 \\
\hline 1111000 & 88.62 & 0.19 & 5.124 \\
\hline 1111100 & 70.34 & 0.18 & 6.25 \\
\hline 111110 & 57.98 & 0.16 & \\
\hline
\end{tabular}

The simulation model of the previous work by [4] is approximated in T-spice under $0.35 \mu \mathrm{m}$ CMOS process. There is seven resistors array and each resistor switches ON/OFF using seven nmos switches. The $32 \mathrm{k}, 30 \mathrm{k}, 25 \mathrm{k}, 22 \mathrm{k}, 20 \mathrm{k}, 18 \mathrm{k}, 14 \mathrm{k}, 10 \mathrm{k}$ resistors are used and the corresponding impedance of the nmos switches are approximately $3.2 \mathrm{k}, 3.0 \mathrm{k}, 2.5 \mathrm{k}, 2 \mathrm{k}, 1.8 \mathrm{k}$, $1.4 \mathrm{k}, 1 \mathrm{k}$, respectively. The operating supply voltage is $2 \mathrm{~V}$. The equivalent resistance of the array changes with the switching of the nmos switches. At different switching conditions (almost $2^{7}$ cases of switching is possible as shown in table 1) specific range of resistance occur which in turn generate specific ranged periodic clocks. Thus, clock generated has discrete range of frequencies as shown in figure 10.

Whereas proposed Analog Field Programmable RC oscillator circuit using FG-pFET generates a continuous and finely tuned frequency ranged clock. With every change (decrease/increase) in floating gate voltage, resistance increase/decrease respectively as tabulated in table 2 .

Table 2: Proposed clock generator using tunable FG-pFET resistor

\begin{tabular}{|c|c|c|c|}
\hline $\begin{array}{c}\text { FG Volt. } \\
\text { (V) }\end{array}$ & Resistance (K) & $\begin{array}{c}\text { Time Period } \\
\text { (us) }\end{array}$ & Frequency (MHz) \\
\hline-0.31762 & 53.37 & 0.2 & 5 \\
\hline-0.26421 & 61.17 & 0.21 & 4.7619 \\
\hline-0.17629 & 75.65 & 0.22 & 4.5454 \\
\hline-0.14521 & 85.92 & 0.231 & 4.3290 \\
\hline-0.08863 & 108.44 & 0.245 & 4.0816 \\
\hline-0.0606 & 118.94 & 0.25 & 4 \\
\hline-0.04759 & 127.45 & 0.258 & 3.8759 \\
\hline-0.00543 & 142.88 & 0.28 & 3.6363 \\
\hline 0.02732 & 154.87 & 0.3 & 3.3333 \\
\hline
\end{tabular}




\begin{tabular}{|c|c|c|c|}
\hline 0.03106 & 176.99 & 0.32 & 3.125 \\
\hline 0.032451 & 182.76 & 0.33 & 3.0303 \\
\hline 0.033759 & 184.35 & 0.34 & 2.9411 \\
\hline 0.036823 & 186.12 & 0.35 & 2.8571 \\
\hline 0.038071 & 190.45 & 0.38 & 2.6315 \\
\hline
\end{tabular}

The frequency range is small $(0.97 \mathrm{MHz}-4.04 \mathrm{MHz})$ or $(1.63 \mathrm{MHz}-5.5 \mathrm{MHz})$ because FGpFET resistor can vary to a certain limit. The floating gate voltage changes from $-0.25 \mathrm{~V}$ to $0.0027 \mathrm{~V}$ at low range of resistances $(45 \mathrm{~K}$ to $130 \mathrm{~K})$ and $\mathrm{FG}$ voltage is -0.31 to 0.03 at high $\mathrm{R}$ (63.37kto180.45k). At low resistance the characteristics are linear as compared to at high resistances. Due to body effect and mobility degradation non linearity occurs when MOS transistor operates in triode region.

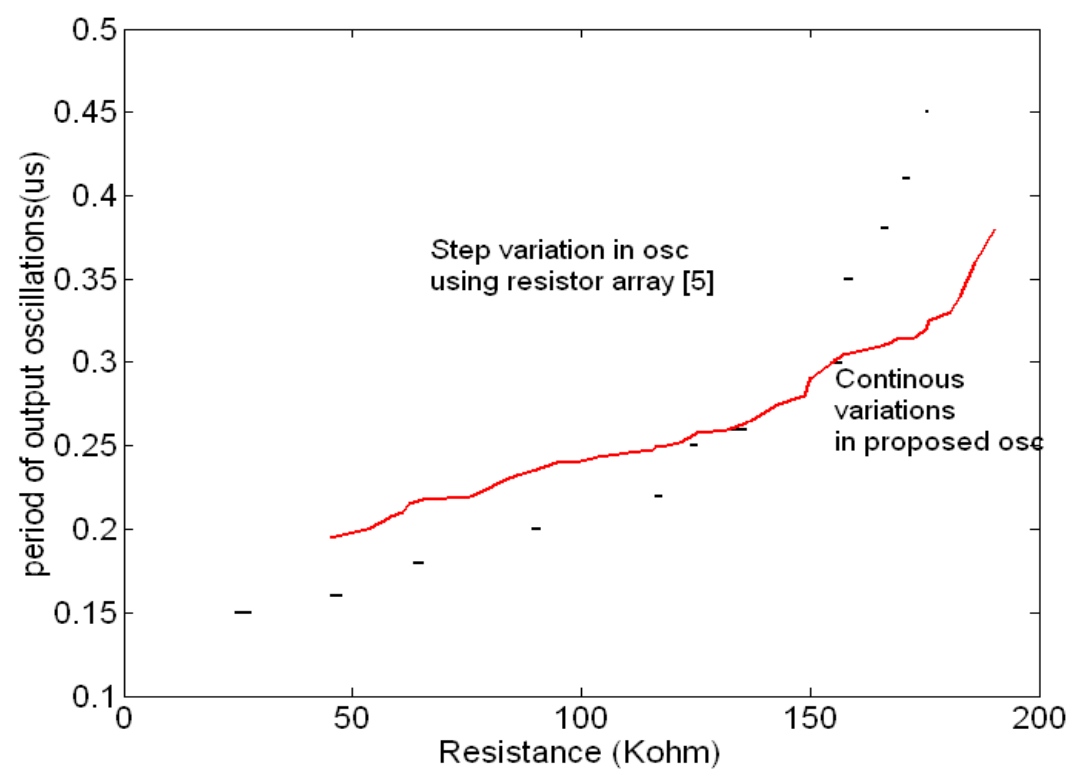

Figure 10: Period variation clock generated in digitally trimmed RC oscillator design inspired

from [5] \& continuous and finely tuned clock generated in proposed clock generator.

However, it gives very high resolution. Least possible change in floating gate voltage which can affect the output clock $=.0003 \mathrm{~V}$. The resolution limit of programmability can be extended to 13-bit as mentioned in paper [1]. Thus, the number of changes in resistance value or trimming can be extended till $\left(2^{\wedge} 13=8192\right.$ cases $)$. Hence, more accurate and fine-tuning of clock can be performed using proposed oscillator design.

\section{Theoretical Approximation of Effective Chip Area}

The chip area of the simulated model inspired from [5] is estimated. The transistor Effective Area $=\min$ poly-silicon width $+2 *($ min poly to contact spacing $)+2 *($ min contact size $)+2 *(\min$ spacing from contact to active area edge). It can be approximated as poly-silicon gate area + $20 \%$ of this area. Thus, effective area of an nmos switch (for say $25 \mathrm{k}$ resistor $\mathrm{w}=2.7 \mathrm{um}, \mathrm{l}=1 \mathrm{um}$ ) $=2.7 \mathrm{um} * 1 \mathrm{um}+0.54 \mathrm{um}=3.24 \mathrm{sq} . \mathrm{um}$. However, in the paper [5] the impedance of the nmos switches is about $0.15 \mathrm{ohm}$, i.e., (high w/l) nmos was considered (hence larger effective area). In 
addition, the resistor $(25 \mathrm{k})$ will consume approximately $35.75 \mathrm{um} * 35.75 \mathrm{um}$ chip area in $0.35 \mathrm{um}$ CMOS technology [18]. Thus, the effective area consumed by the resistors array comes out to be approximately 10224.5 sq.um. Hence, the effective chip area consumed by the switching resistor array is 10259.48 sq.um.

However, in the proposed RC oscillator circuit design two layers of poly-silicon are used. The effective area of the floating gate PFET synapse [19] is approximately $110 \mathrm{um} * 93 \mathrm{um}$ when the floating gate PFET synapse is generated in 0.5um CMOS process technology. The area occupied by the capacitor (double poly layer) affect the total area of the circuit design. In addition, this Floating gate PFET synapse will implement in 0.35 um CMOS technology. Therefore, the chip area occupied by the floating gate PFET will reduce further.

\section{ON-Chip Programmability}

The period of the output clock depends on floating-gate voltage, as shown in figure11. With fixed $\mathrm{W}$ and $\mathrm{L}$ specifications of floating-gate pFET, the circuit can be trimmed by just varying floating gate voltage of the floating gate pFET (by varying charge at the floating-gate) fabricated as a part of proposed design of clock generator. Thus, the generation of finely tuned frequency clock can be field programmable by simple tweaking charge at gate voltage of transistors.

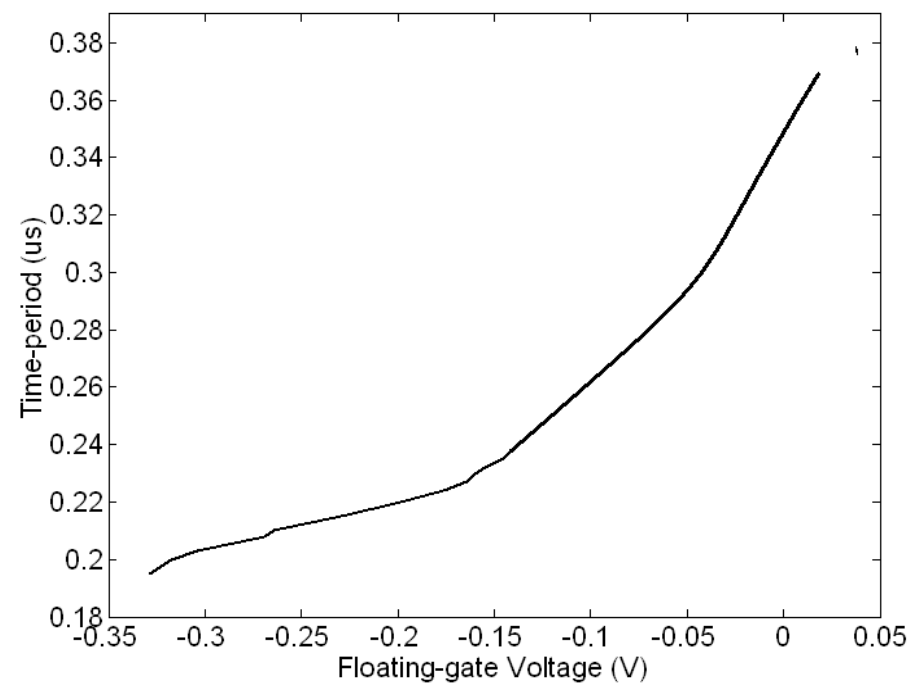

Figure 11: Variations in Clock period with change in floating gate voltage of tunable FG-pFET resistor in the proposed circuit.

\section{Topology to Widen the Clock RANGe}

The proposed circuit generates very highly precise and continuous ranged clock as mentioned in last sections. However the range in which clock period can be programmed is small because floating gate voltage of FG-pFET resistor can vary to a certain limit. Thus, to increase this range place transistors with different W/L values in parallel with FG-pFET in the proposed circuit (figure 4.) with the common floating gate. For example, two transistor with w/l (2/1) and (2/0.8) are used independently then, range of resistances obtained respectively are (45K to $130 \mathrm{~K})$ and 
(63.37kto180.45k); and hence clock obtained are (0.97MHz-4.04MHz) and (1.63MHz-5.5MHz) respectively. When placed parallel with common floating gate, the clock can be generated with frequency variation in the range between $0.97 \mathrm{MHz}-5.5 \mathrm{MHz}$. As shown in Figure 12 the range of clock can be increased further by increasing more number of transistors at common floating gate.

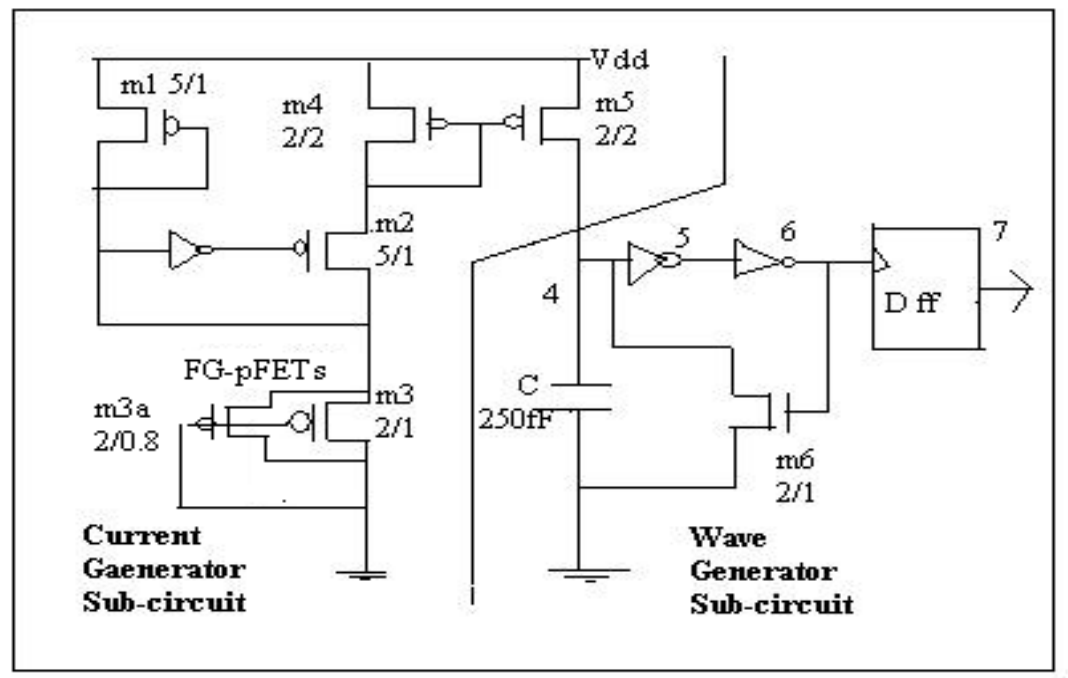

Figure 12: Circuit Diagram showing Topology to extend the frequency range of output clock

\section{TEMPERATURE STABILITY}

TABLE 3: Variations with Temperature

\begin{tabular}{|c|c|c|}
\hline Temperature $\left({ }^{\circ} \mathbf{C}\right)$ & Time period(us) & Frequency(MHz) \\
\hline 0 & 0.280982 & 3.56 \\
\hline 10 & 0.279235 & 3.58 \\
\hline 23 & 0.268301 & 3.77 \\
\hline 25 & 0.265236 & 3.78 \\
\hline 27 & 0.264132 & 3.84 \\
\hline 30 & 0.259952 & 3.98 \\
\hline 60 & 0.250887 & 4.16 \\
\hline 80 & 0.240421 & \\
\hline
\end{tabular}


In proposed design the variation of FG-pFET resistor values varies with temperature as shown in figure 13. In addition, the frequency and time period change with temperature variation from $0{ }^{\circ} \mathrm{C}$ to $80{ }^{\circ} \mathrm{C}$ is shown in Table 3 . The period varies at rate of $0.507 \mathrm{~ns} /{ }^{\circ} \mathrm{C}$.

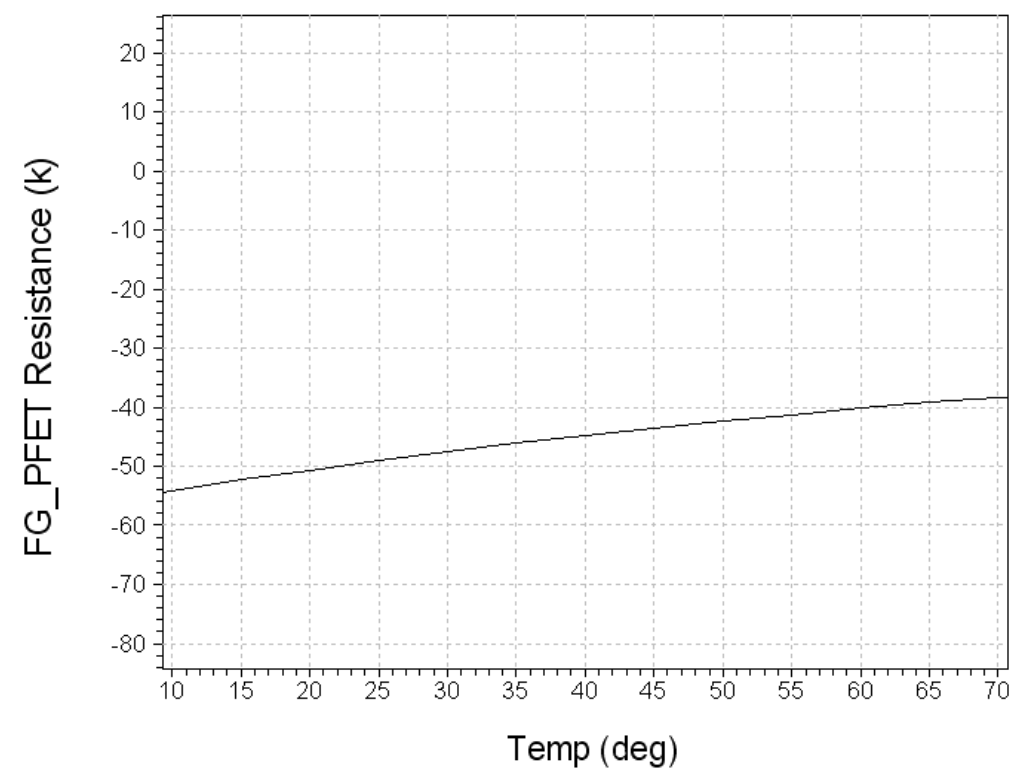

Figure 13: Plot representing FG-pFET resistance variation with change in temperature (about $\left.0.1875 \mathrm{~K} /{ }^{\circ} \mathrm{C}\right)$

The temperature dependence of the FG-pFET can be obtained using equation 4 and 5:

$$
R_{F G_{-} P F E T}=\frac{L}{\mu C_{o x} W\left(V_{g s}-V_{t h}\right)}
$$

The temperature dependence of $\mu$ and $V_{T}$ can expressed as $\mu=\mu_{n o}\left(T / T_{0}\right)^{-m}$ and $V_{T}=V_{T_{0}}{ }^{-}$ $\alpha_{\mathrm{VT}}\left(\mathrm{T}-\mathrm{T}_{0}\right)$, where $\mathrm{T}_{\mathrm{o}}$ is the reference temperature, and $\mathrm{m}$ is the positive constant that ranges from 1.5 to 2 , and $\mu_{\text {no }} \& \mathrm{~V}_{\mathrm{T} 0}$ are the temperature independent parameters. Also $\alpha_{\mathrm{VT}}$ is in the range of 0.5 to $4 \mathrm{mV} /{ }^{\circ} \mathrm{C}[15]$. Hence, the temperature coefficient of the FG-pFET can be expressed as

$$
\frac{1}{R} \frac{\delta R}{\delta Y}=-\frac{1}{\mu} \frac{\delta \mu_{n o}}{\delta T}+\frac{1}{V_{g}-V_{T}} \frac{\delta V_{T}}{\delta T}=\frac{m}{T}-\frac{\alpha_{V T}}{V_{g}-V_{T}}
$$

where, $\frac{1}{\mu} \frac{\delta \mu_{n o}}{\delta T}=-\frac{1}{m} \& \frac{\delta V_{T}}{\delta T}=-\alpha_{V T}$. As a result, the temperature coefficient of the FGPFET can be tuned by altering the effect of $\alpha_{\mathrm{VT}}$ through the use of $\mathrm{V}_{\mathrm{g}}$. For desired temperature, $\mathrm{T}_{\mathrm{d}}$, and $V_{g}=\frac{\alpha_{V T}}{V_{g}-V_{T}} T_{d}+V_{T}$ the temperature coefficient of the FG-pFET can be set to zero at $\mathrm{T}_{\mathrm{d}}$. Hence, at specific temperature by adjusting the floating gate voltage temperature coefficient of the FG-pFET can be tuned to zero 


\section{ConClusion}

The floating gate transistor using T-Spice, $0.35 \mu \mathrm{m}$ CMOS process, was successfully implemented as an efficient and accurate RC Oscillator circuit. FG-pFET resistors easily achieve high and precise (13bit) resistance values. FG-pFET resistor used in the circuit generates continuous and linear frequency range $(1.63-5.5 \mathrm{MHz}$ at FG-pFET's $\mathrm{R}=110-500 \mathrm{~K}$ or $0.95-4.04 \mathrm{MHz}$ at FG-pFET's $\mathrm{R}=63.87-183.84 \mathrm{~K}$ ).By switching multiple FG-pFET (with different $\mathrm{W} / \mathrm{Ls}$ ) at common floating gate, frequency range can be increased. With tweaking charges at the floating gate of FG-pFET, the frequency of the square wave can be finely and accurately tuned. The 13 bit of programming resolution can be obtained. It can also be operated at $1 \mathrm{v}$ supply voltage.

The circuit is adaptive to the change in temperature. By programming the FG-pFET the temperature coefficient can be tuned to zero value at any specific temperature. The size of the proposed circuit was relatively reduced when compared to other on-chip discrete trimming circuits. The proposed clock generator produces analog, highly precise and widely tuned square waveform which can find its application in wired or wireless network equipment which requires clock for highly accurate and high speed data transmission.

Table 4:Comparative Analysis of latest clock generators (RC oscillators)

\begin{tabular}{|c|c|c|c|c|c|}
\hline Parameters & Proposed osc & S.Yu, et.al[5] & $\begin{array}{l}\text { C.Ghidini, } \\
\text { et.al.[6] }\end{array}$ & J.H Choi[7] & $\begin{array}{c}\text { S.K.Kim, } \\
\text { et.al [8] }\end{array}$ \\
\hline Technique & FG-PFET & $\begin{array}{l}\text { On-chip } \\
\text { resistors array }\end{array}$ & $\begin{array}{c}5 \& 3 \text { bit } \mathrm{C} \& \mathrm{R} \\
\text { arrays }\end{array}$ & $\begin{array}{l}\text { 4-bit weighted- } \\
\text { capacitors }\end{array}$ & $\begin{array}{c}\text { OTFT } \\
\text { transistors }\end{array}$ \\
\hline Technology & $0.35 \mathrm{um}$ & $0.5 \mathrm{um}$ & $0.25 \mathrm{um}$ & $0.28 \mathrm{um}$ & $\begin{array}{c}\text { Not } \\
\text { mentioned }\end{array}$ \\
\hline $\begin{array}{l}\text { Min supply } \\
\text { Voltage }\end{array}$ & $1.8 \mathrm{v}$ & $4.5 \mathrm{v}$ & $2 \mathrm{v}$ & $1.8 \mathrm{v}$ & $-40 v$ \\
\hline $\begin{array}{l}\text { Frequency } \\
\text { Range }\end{array}$ & $\begin{array}{l}5.5 \mathrm{MHz}- \\
1.63 \mathrm{MHz}\end{array}$ & $\begin{array}{l}\text { 2.22MHz- } \\
6.67 \mathrm{MHz}^{2}\end{array}$ & $\begin{array}{l}30 \mathrm{MHz}- \\
36 \mathrm{MHz}\end{array}$ & $\begin{array}{l}1.6 \mathrm{MHz}- \\
2.4 \mathrm{MHz}\end{array}$ & $140 \mathrm{KHz}$ \\
\hline $\begin{array}{l}\text { Oscillations } \\
\text { behaviour }\end{array}$ & Continous & Discrete & Discrete & Discrete & Discerte \\
\hline $\begin{array}{l}\text { Frequency } \\
\text { variation }\end{array}$ & $0.507 \mathrm{~ns} /{ }^{\circ} \mathrm{C}$ & $\begin{array}{c}0.015 \mathrm{~ns} /{ }^{\circ} \mathrm{C} \\
(0.5 \%)\end{array}$ & $2 \%$ & Not mentioned & $\begin{array}{c}\text { Not } \\
\text { mentioned }\end{array}$ \\
\hline $\begin{array}{c}\text { Temperature } \\
\text { range }\end{array}$ & $0^{\circ} \mathrm{C}-80^{\circ} \mathrm{C}$ & $0^{\circ} \mathrm{C}-80^{\circ} \mathrm{C}$ & $0^{\circ} \mathrm{C}-80^{\circ} \mathrm{C}$ & Not mentioned & $\begin{array}{c}\text { Not } \\
\text { mentioned }\end{array}$ \\
\hline On-chip size & $(110 \mathrm{um} \times 93 \mathrm{um})^{5}$ & 130um $\times 145$ um & 444um×280um & 240um $\times 130 u m$ & $\begin{array}{c}\text { Not } \\
\text { mentioned }\end{array}$ \\
\hline $\begin{array}{l}\text { Programming } \\
\text { resolution }\end{array}$ & $\begin{array}{c}13 \text {-bit } \\
\left(2^{\wedge} 13=8192\right)[13]\end{array}$ & 7 bit $\left(2^{\prime}=128\right)$ & 8-bit & 4-bit & $\begin{array}{c}\text { Not } \\
\text { mentioned }\end{array}$ \\
\hline
\end{tabular}

${ }^{1}$ simulated results

${ }^{2}$ from simulated model on 0.35 um CMOS process

${ }^{3}$ Theoretically estimate

The proposed clock generator is being compared with the latest clock generators (RC oscillators) and noted in table IV. The proposed clock generator provides continuous and finely tuned (13bit resolution) clock. Comparable frequency range which can be extendable using 
International journal of VLSI design \& Communication Systems (VLSICS) Vol.1, No.3, September 2010

topology as explained before. Comparable clock period variation with temperature change which too can be compensated.

\section{ACKNOWLEDGMENT}

The authors wish to thank funding agency MHRD and DST, Govt of India for the financial support for this work.

\section{REFERENCES}

[1] Y. L. Wong, M.H Cohen, P. A. Abshire,” A $1.2 \mathrm{GHz}$ adaptive floating gate comparator with 13-bit resolution”, ISCAS 2005, pg 6146-49, Vol.6, IEEE, 2005.

[2] B. B. Bhattacharya, "A unified approach to the realization of canonic RC-active, single as well as variable, frequency oscillators using operational amplifiers," Franklin Institute, Journal (ISSN 0016-0032), vol. 317, pg: 413-439. Sponsorship: Natural Sciences and Engineering Research Council, June 1984.

[3] K.N. Salama, A.M. Soliman," Novel oscillators using the operational transresistance amplifier," Microelectronics Journal, Vol. 31, pg. 39-47, 2000.

[4] S.Yu, Y.Chen, W. Guo, X. Che, "A digital-trim controlled on-chip RC oscillator ," Circuit and systems 2001, MWSCAS 2001, proceedings of the $44^{\text {th }}$ IEEE 2001 Midwest symposium, Vol. 2, pg 882-885, 14-17 Aug, 2001.

[5] C. Ghidini, J .G. Aranda, D. Gerna ,K .Kelliher ,’A digitally programmable on-chip RC oscillator in 0.25 $\mu \mathrm{m}$ CMOS logic process”, ISCAS 2005, IEEE International Symposium, Vol. 1. pg: 400403, 23-26 May, 2005.

[6] J.H Choi, "Minimization of parasitic effects of an accurate 2-MHz RC oscillator for low voltage and low power applications," pages 219-223, IEEE, sept,2005.

[7] S.K.Kim, S.B.Kwon,B.S.Bae,’Organic Thin Film Transistor RC Oscillator,” Symposium Proceedings Series, Materials Research Society, Vol. 1003E, pg. 434-436, 2007.

[8] A. Hastings, The art of analog layout”, Prentice-Hall Inc., 2000.

[9] P. R. Gray, R. G. Meyer, P. J. Hurst, S. H. Lewis, "Analysis and Design of Analog Integrated Circuits", 4th Ed. John Wiley and Sons, 2005. 
International journal of VLSI design \& Communication Systems (VLSICS) Vol.1, No.3, September 2010

[10] D. Graham, E. Farquhar, B. Degnan, C. Gordon, and P. Hasler:, "Indirect programming of floatinggate transistors," Proceedings of the International Symposium on Circuits and Systems, vol. 1, pp. 2172-2175, May 2005.

[11] K. Nay and A. Budak, "A voltage-controlled resistance with wide dynamic range and low distortion,” IEEE Trans. on Circuit and Systems, vol. 30, pg. 770-772, Oct, 1983.

[12] W. R. Patterson and F. S. Shoucair, "Harmonic suppression in unbalanced analogue MOSFET circuit topologies using body signals,” Electronic Letters, vol. 25, pg. 1737-1739, Dec.1989.

[13] E. Ozalevli, "Exploiting floating gate transistor properties in analog and mixed signal circuit design," Thesis work, Electrical and Commuter Engineering, Georgia Institute of Technology, Atlanta, Dec 2006.

[14] K. Rahimi, C. Diorio, C. Hernandez, M. D. Brockhausen, , “A Simulation model for floating gate MOS synapse transistors," Vol 14, pg. 1354-1367, IEEE educational Activities Department, Dec 2006.

[15] Y. P Tsividis, Operation and Modeling of the MOS transistor, New York, McGraw-Hill Companies, Inc., 1987.

[16] Axel Thomsen and Martin A. Brooke, "A Floating-Gate MOSFET with Tunneling Injector Fabricated Using a Standard Double-Polysilicon CMOS Process,” IEEE Electron Device Letters, vol. 12, no. 3, pp. 111-113, March 1991.

[17] R. Suri, C. M Markan, Threshold Trimming based design of a CMOS Operational Amplifier, $19^{\text {th }}$ International Conference on VLSI Design (VLSI Design 2006), Hyderabad, pg 717-720, 3-7, Jan, 2006.

[18] L. R. Carley, Trimming Analog circuits using floating-gate analog MOS memory, IEEE Journal of Soli-State Circuits, vol.24, pp. 1569-1574, Dec, 1989.

[19] C. Diorio, P. Hasler, B. A Minch and C. Mead, A single-transistor silicon synapses, IEEE Transaction on Electron Devices, vol. 43, no.11,pg 1972-1980, 1996.

[20] A. Josan, K Kumar, C. M Markan, Design of Multiple output field programmable voltage reference using floating gate transistors, $13^{\text {th }}$ VDAT conference, Bangalore, 8-10July, 2009. 


\section{Authors:}

Garima Kapur is currently perusing research in VLSI Design Lab, Department of Physics and Computer Science, Dayalbagh Educational Institute, Agra, India. After completing M. Tech in VLSI and Embedded System Design from MACT, Bhopal, INDIA. Her interests are in applications of floating gate transistors as tunable resistor. She is a student member of IEEE and VLSI society of India.

Dr. C. M. Markan is an Associate Professor in Department of Physics and Computer Science, Dayalbagh Educational Institute, Dayalbagh, Agra, India. His areas of interests are in Neuromorphic and Adaptable Analog VLSI, Virtual Laboratories. He is a member of IEEE, Systems Society of India, and International Association of Online Engineering.

Prof. V. Prem Pyara is currently professor in the Department of Electrical Engineering, Dayalbagh Educational Institute, Dayalbagh, Agra, India. His current areas of interest are Electronics and Signal Processing.
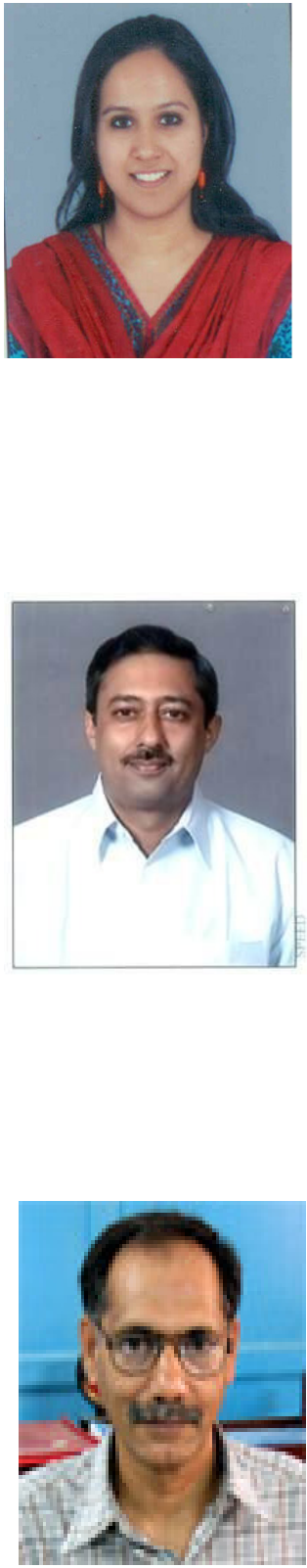